\title{
The Concomitance of Cervical Spondylosis and Adult Thoracolumbar Spinal Deformity
}

\author{
Michael H. Weber ${ }^{1} \quad$ C. H. Hong ${ }^{2}$ William W. Schairer ${ }^{2}$ Steven Takemoto ${ }^{2}$ Serena S. Hu ${ }^{2}$ \\ ${ }^{1}$ Division of Orthopaedic Surgery, McGill University, Montreal, \\ Quebec, Canada \\ 2 Department of Orthopaedic Surgery, University of California, \\ San Francisco, California, United States \\ Address for correspondence Serena S. Hu, MD, Department of \\ Orthopaedic Surgery, Stanford University Hospital and Clinics, 450 \\ Broadway Street, Redwood City, CA 94063, United States \\ (e-mail: serena.hu@stanford.edu).
}

Evid Based Spine Care J 2014;5:6-11.

\begin{abstract}
Keywords

- cervical spondylosis

- scoliosis

- myelopathy

- adult spine deformity

- administrative claims data
\end{abstract}

Study Design Retrospective cross-sectional study.

Clinical Question What is the prevalence of cervical spondylosis (CS) and thoracolumbar (TL) spinal deformity in an administrative database during a 4-year study period? Is the prevalence of CS or TL deformity higher in patients who have the other spine diagnosis compared with the overall study population? Are patients with both diagnoses more likely to have undergone spine surgery?

Patients and Methods An administrative claims database containing 53 million patients with either Medicare (2005-2008) or private payer (2007-2010) insurance was used to identify patients with diagnoses of CS and/or TL deformity. Disease prevalence between groups was compared using a $\chi^{2}$ test and reported using prevalence ratios (PR).

Results The prevalence of CS was higher in patients with TL deformity than without TL deformity, for both Medicare $(P R=2.81$ ) and private payer $(P R=1.79)$. Similarly, the prevalence of TL deformity was higher in patients with CS than without CS for both Medicare $(P R=3.19)$ and private payer $(P R=2.05)$. Patients with both diagnoses were more likely to have undergone both cervical (Medicare, $P R=1.44$; private payer, $\mathrm{PR}=2.03$ ) and $\mathrm{TL}$ (Medicare, $\mathrm{PR}=1.68$; private payer, $\mathrm{PR}=1.74$ ) spine fusion. All comparisons were significant with $p<0.0001$.

Conclusions Patients with either CS or TL deformity had a higher prevalence of the other spinal diagnosis compared with the overall disease prevalence in the study population. Patients with both diagnoses had a higher prevalence of having spine surgery compared with patients with only one diagnosis. More studies to identify a causal mechanism for this relationship are warranted.

\section{Study Rationale and Context}

Cervical spondylosis (CS) is common in the elderly and usually asymptomatic, but it can be severely disabling to many patients. Patients with scoliosis often have neck pain, ${ }^{1}$ possibly caused by altered cervical alignment after spine fusion, ${ }^{2,3}$ but this relationship has yet to be quantified. This

received

March 16, 2013

accepted after revision

December 11, 2013

study evaluated the prevalence of CS in patients with thoracolumbar (TL) spinal deformity.

\section{Clinical Question}

What is the prevalence of CS and TL deformity as recorded in two medical claims databases over a 4-year study period?

(c) 2014 Georg Thieme Verlag KG Stuttgart · New York
DOI http://dx.doi.org/ 10.1055/s-0034-1368668. ISSN 1663-7976. 
What is the prevalence of CS in patients with TL deformity; what is the prevalence of TL deformity in patients with CS? What is the prevalence of spine surgery in patients with one or both diagnoses of CS and TL deformity?

\section{Patients and Methods}

\section{Study Design}

This study is a retrospective cross-sectional study.

\section{Inclusion Criteria}

All patients with a medical claim during the study time period, listed in the Medicare (2005-2008) or private (2007-2010) insurance databases.

\section{Exclusion Criteria}

No patients were excluded because this was a population study.
Patient population and selection criteria are as follows (see - Fig. 1):

- Patients were identified using the PearlDiver administrative claims database, a nationwide repository of $100 \%$ of inpatient and outpatient claims and 5\% physician claims for 38 million patients with Medicare and 15 million patients with private payer insurance (see the online supplementary material).

- International Classification of Diseases 9 (ICD-9) diagnosis codes were used to identify patients with diagnoses of CS and/or TL deformity.

- The prevalence of cervical and TL surgery was assessed using ICD-9-CM procedure codes for spine surgery.

\section{Outcomes and Prognostic (Risk) Factors to be Evaluated}

- Prevalence of CS and TL deformity in study population.

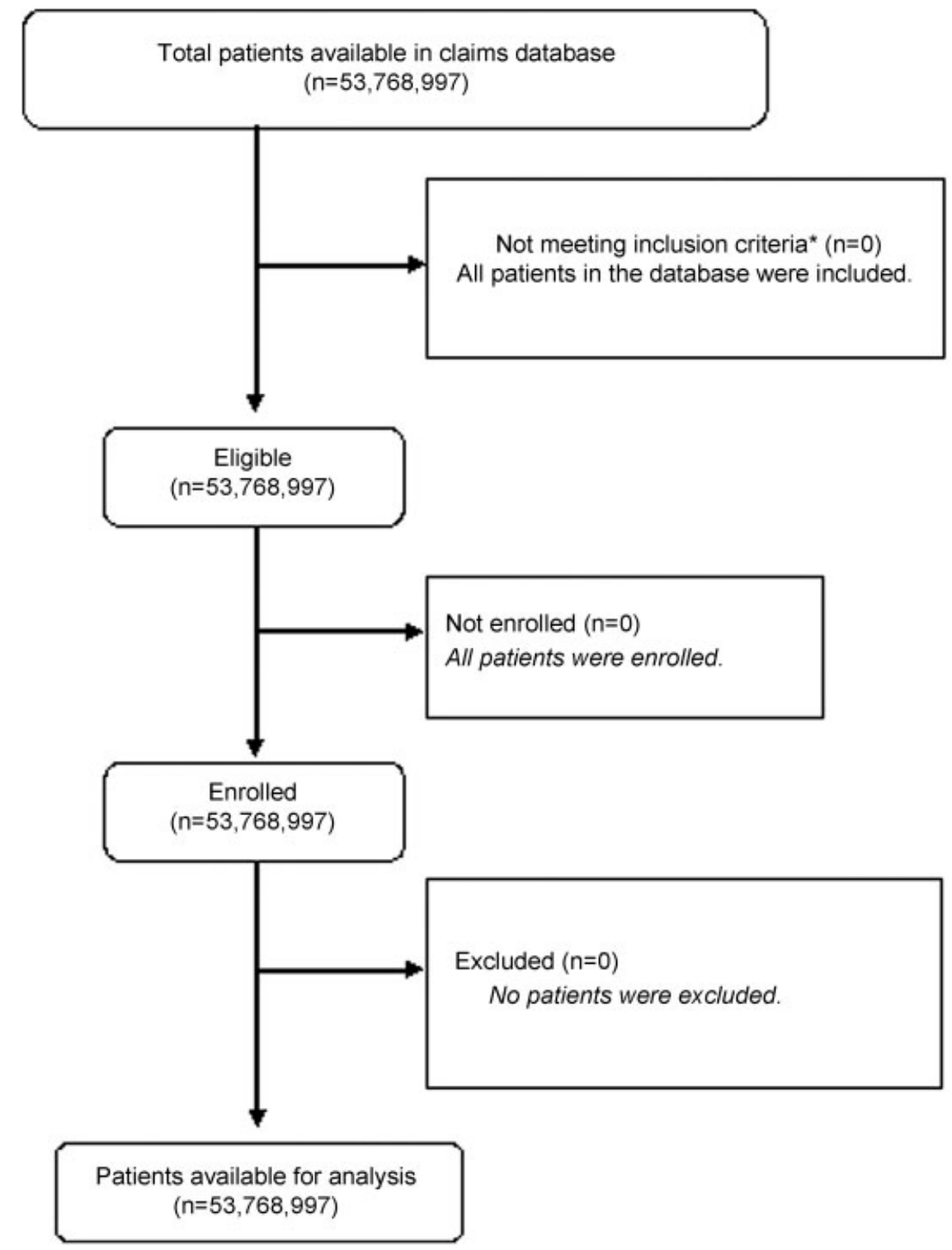

Fig. 1 Patient sampling and selection. 
Table 1 Patient characteristics

\begin{tabular}{|c|c|c|}
\hline & $\begin{array}{l}\text { Medicare insurance } \\
N=38,492,730(\%)\end{array}$ & $\begin{array}{l}\text { Private payer insurance } \\
N=15,276,267(\%)\end{array}$ \\
\hline \multicolumn{3}{|l|}{ Patient characteristics } \\
\hline Female & 56.1 & 53.7 \\
\hline Age $<65$ y & 12.7 & 98.8 \\
\hline Age $>65 y$ & 87.3 & 1.2 \\
\hline Cervical spondylosis & 8.5 & 16.0 \\
\hline Thoracolumbar spinal deformity & 2.4 & 2.2 \\
\hline Both diagnoses & 0.6 & 0.5 \\
\hline
\end{tabular}

- Comparing prevalence of CS diagnoses in patients with and without TL deformity.

- Comparing prevalence of TL deformity in patients with and without CS.

- Comparing prevalence of cervical and/or TL spine surgery in patients with one diagnosis versus both diagnoses.

\section{Analysis}

- Comparisons were performed with a Pearson $\mathrm{X}^{2}$ test and reported using prevalence ratios (PR).

- A $p$ value less than 0.05 was considered statistically significant.

Additional methodological and technical details are provided in the Web Appendix.

\section{Results}

\section{Single Diagnosis}

- The prevalence of CS in the study population was $8.5 \%$ Medicare and $16.0 \%$ private payer (-Table $\mathbf{1}$ ).

- In patients with CS, cervical spine surgery was performed in $4.3 \%$ Medicare and $2.2 \%$ private payer.

- The prevalence of TL deformity was $2.4 \%$ Medicare and $2.2 \%$ private payer.
- In patients with TL deformity, TL spine surgery was performed in 3.1\% Medicare and 3.6\% private payer.

\section{Multiple Diagnoses}

- The overall prevalence of patients with both diagnoses was relatively uncommon at $0.6 \%$ in Medicare patients and $0.5 \%$ in private payer patients.

- The prevalence of CS was elevated in patients with TL deformity compared to without TL deformity: 22.9 versus $8.2 \%$ for Medicare $(\mathrm{PR}=2.81 ; p<0.0001)$ and 28.0 versus $15.7 \%$ for private payer $(\mathrm{PR}=1.79 ; p<0.0001)(-$ Table 2$)$.

- Elevated prevalence of cervical spine surgery in patients with both CS and TL deformity than CS alone: 6.0 versus $4.1 \%$ for Medicare $(\mathrm{PR}=1.44 ; p<0.0001)$ and 4.2 versus $2.1 \%$ for private payer $(\mathrm{PR}=2.03 ; p<0.0001)(-$ Table 3$)$.

- The prevalence of TL deformity was elevated in patients with CS than without CS: 6.6 versus $2.1 \%$ for Medicare $(\mathrm{PR}=3.19 ; p<0.0001)$ and 3.8 versus $1.9 \%$ for private payer $(\mathrm{PR}=2.05 ; p<0.0001)$.

- Elevated prevalence of TL spine surgery in patients with both TL deformity and CS than with TL deformity alone: 4.6 versus $2.7 \%$ for Medicare $(\mathrm{PR}=1.68 ; p<0.0001)$ and 5.2 versus $3.0 \%$ for private payer $(\mathrm{PR}=1.74 ; p<0.0001)$ (-Table 4).

Table 2 Concomitant spine diagnoses

\begin{tabular}{|l|l|l|}
\hline & $\begin{array}{l}\text { Medicare } \\
N=38,492,730\end{array}$ & $\begin{array}{l}\text { Private payer } \\
N=15,276,267\end{array}$ \\
\hline CS (\%) & $n=3,276,261(8.5)$ & $n=2,436,959(16.0)$ \\
\hline Patients without TL deformity (\%) & $3,060,810(8.2)$ & $2,343,236(15.7)$ \\
\hline Patients with TL deformity (\%) & $215,451(22.9)$ & $93,723(28)$ \\
\hline Prevalence ratio (95\% Cl) & $2.81(2.80-2.82)^{\mathrm{a}}$ & $1.79(1.78-1.80)^{\mathrm{a}}$ \\
\hline TL deformity (\%) & $n=941,421(2.4)$ & $n=334,492(2.2)$ \\
\hline Patients without CS (\%) & $725,970(2.1)$ & $240,769(1.9)$ \\
\hline Patients with CS (\%) & $215,451(6.6)$ & $93,723(3.8)$ \\
\hline Prevalence ratio (95\% Cl) & $3.19(3.18-3.20)^{\mathrm{a}}$ & $2.05(2.04-2.07)^{\mathrm{a}}$ \\
\hline
\end{tabular}

Abbreviations: $\mathrm{Cl}$, confidence interval; $\mathrm{CS}$, cervical spondylosis; $\mathrm{TL}$, thoracolumbar.

andicates $p<0.001$. 
Table 3 Prevalence of cervical spine surgery in patients with cervical spondylosis

\begin{tabular}{|l|l|l|}
\hline & $\begin{array}{l}\text { Medicare } \\
N=3,276,261\end{array}$ & $\begin{array}{l}\text { Private payer } \\
N=2,436,959\end{array}$ \\
\hline Cervical spine surgery (\%) & $n=139,452(4.3)$ & $n=52,514(2.2)$ \\
\hline Patients without TL deformity (\%) & $126,589(4.1)$ & $48,574(2.1)$ \\
\hline Patients with TL deformity (\%) & $12,863(6.0)$ & $3,940(4.2)$ \\
\hline Prevalence ratio (95\% Cl) & $1.44(1.42-1.47)^{\mathrm{a}}$ & $2.03(1.96-2.09)^{\mathrm{a}}$ \\
\hline
\end{tabular}

Abbreviations: $\mathrm{Cl}$, confidence interval; $\mathrm{TL}$, thoracolumbar.

andicates $p<0.001$.

Table 4 Prevalence of thoracolumbar spine surgery in patients with thoracolumbar deformity

\begin{tabular}{|l|l|l|}
\hline & Medicare & $\begin{array}{l}\text { Private payer } \\
N=334,492\end{array}$ \\
\hline Thoracolumbar spine surgery (\%) & $n=29,490(3.1)$ & $n=12,102(3.6)$ \\
\hline Patients without CS (\%) & $19,663(2.7)$ & $7,212(3.0)$ \\
\hline Patients with CS (\%) & $9,827(4.6)$ & $4,890(5.2)$ \\
\hline Prevalence ratio (95\% Cl) & $1.68(1.64-1.72)^{\mathrm{a}}$ & $1.74(1.68-1.80)^{\mathrm{a}}$ \\
\hline
\end{tabular}

Abbreviations: $\mathrm{Cl}$, confidence interval; $\mathrm{CS}$, cervical spondylosis.

andicates $p<0.001$.

\section{Discussion}

\section{Strengths}

- Very large patient cohort.

- Diverse population of patients with either Medicare or private health insurance.

\section{Limitations}

- Retrospective analysis that describes correlation but cannot attribute cause and effect.

- Nonvalidated administrative data sets for billing purposes may lack important clinical accuracy.

- Could not track patients from private insurance to Medicare database, thus could be counted twice if they became Medicare eligible.

- Could not exclude children due to limitations of database coding.

\section{Clinical Relevance and Impact}

- Prior studies have shown a high incidence of neck pain in patients with scoliosis, with increased incidence in TL fusion patients.

- This is the first study to quantify the increased prevalence of CS and TL spinal deformity in patients who already have one diagnosis.

- Physicians may wish to screen for symptomatic CS in patients who present with adult TL spinal deformity (and vice versa).

- Patients with both diagnoses were more likely to receive spine surgery, but it is unknown how having both diagnoses may affect patient's expected satisfaction and clinical outcome.
- Future research with direct medical record review could establish the timing and severity of developing CS after the diagnosis of TL spinal deformity (and vice versa).

\section{Case Study}

A 63-year-old man presented for surgical evaluation of worsening back pain and radiating leg pain due to degenerative scoliosis deformity of his lumbar spine. Clinical exam showed mildly decreased strength on his right lower extremity, and was also notable for a wide-based gait, very brisk reflexes in all extremities, and a positive Hoffman sign bilaterally. On further questioning, the patient denied neck pain, but did acknowledge worsening dexterity in both hands. Because of concern for myelopathy, a cervical magnetic resonance image was obtained and showed CS from C5 to C7, with hypertrophic ligamentum flavum and a disc osteophyte complex anteriorly (-Fig. 2). The patient underwent anterior cervical discectomy and fusion from C5 to C7 with no complications. Six months after the operation, he had fully recovered upper extremity dexterity, and had also felt like he had improved lower extremity strength. However, he continued to have back and radiating leg pain. Full-length spinal radiographs showed a left lumbar curve from L1 to L5 (- Fig. 3). The patient underwent a staged posterior spinal fusion from T10 to S1 with interbody cages from L2 to S1 with no complications. At 1-year postoperatively, the patient was asymptomatic with no back or leg pain.

This case demonstrates a patient who presented with a complaint related to degenerative spinal deformity but was found to also have CS causing myelopathy. This highlights the importance of a complete neurological exam in patients who present with spinal deformity, as they are at increased risk of also having symptomatic CS. 

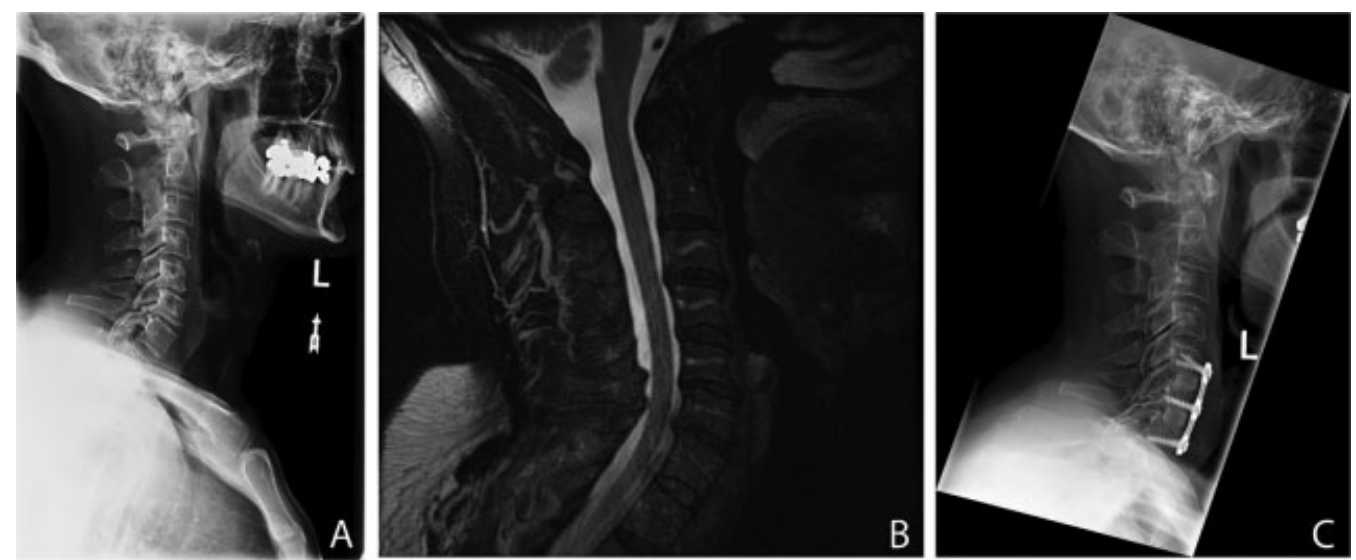

Fig. 2 (A) Preoperative lateral cervical X-ray and (B) sagittal cervical magnetic resonance imaging showing degenerative spondylosis with spinal cord compression. (C) Postoperative lateral cervical X-ray showing solid fusion from C5 to C7. The patient fully recovered dexterity and had improved lower extremity strength after the operation.
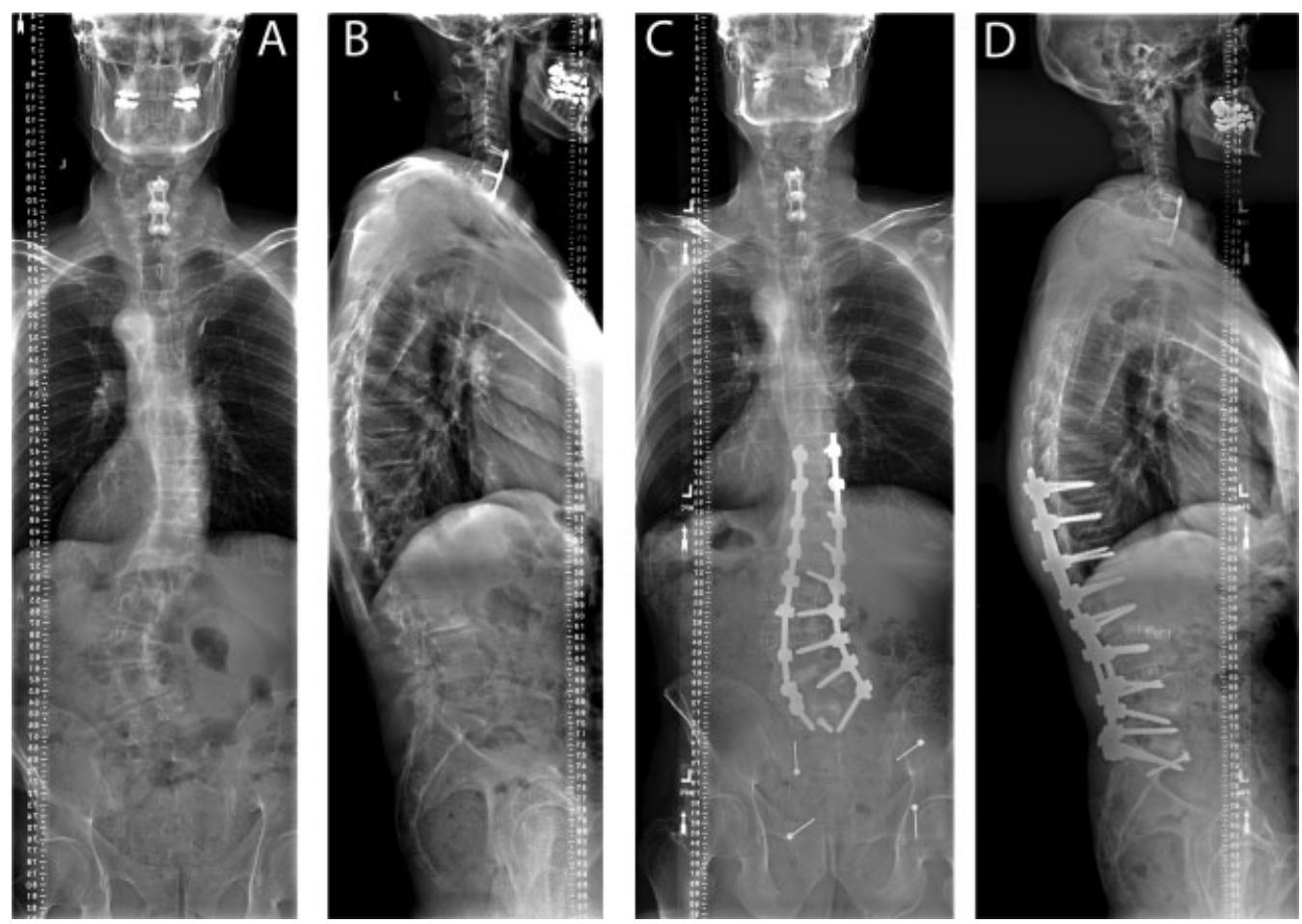

Fig. 3 (A) Preoperative anteroposterior and (B) lateral full spine X-rays showing left degenerative lumbar scoliosis. (C, D) The patient successfully underwent a posterior spinal fusion with complete resolution of his back and leg pain.

This study received Institutional Review Board approval by the Committee on Human Research at the University of California, San Francisco, United States.

\section{Summary and Conclusions: Key Points}

- Patients with a diagnosis of CS or TL spinal deformity are two to three times more likely also to have the other diagnosis.

- Patients with both diagnoses are more likely to have undergone spine surgery.

\section{Funding Disclosure}

No funds were received for the support of this study.

\section{References}

1 Moskowitz A, Moe JH, Winter RB, Binner H. Long-term follow-up of scoliosis fusion. J Bone Joint Surg Am 1980;62(3):364-376

2 Edgar MA, Mehta MH. Long-term follow-up of fused and unfused idiopathic scoliosis. J Bone Joint Surg Br 1988;70(5):712-716

3 Hilibrand AS, Tannenbaum DA, Graziano GP, Loder RT, Hensinger RN. The sagittal alignment of the cervical spine in adolescent idiopathic scoliosis. J Pediatr Orthop 1995;15(5):627-632 


\section{Editorial Perspective}

Our reviewers unanimously congratulated Weber et al for what they labeled a "benchmark study." In addition to its findings, this article deserves our attention for its conceptual and methodological approaches.

The question of a possible interrelation of thoracolumbar deformities and cervical spondylosis has never been conclusively addressed. Just now, after several decades of concentrating on measuring and classifying thoracolumbar deformities, the spinal deformity community is beginning to take a more expansive view of the global spinal alignment inclusive of the cervical spine. From refinements in cervical radiographic alignment measurements to improved understanding of the spatial interrelationship of thoracolumbar and cervical spine columns, and the causal interdependence of cervical spine deformity on the evolution of cervical spondylotic myelopathy, there is a rapidly emerging body of literature on this topic. ${ }^{1-3}$ Undoubtedly, this article, with its massive study cohort of well over 53 million patients, will increase the awareness of our spine community regarding the cervical spine in dealing with thoracolumbar deformities and vice versa.

From a methodological perspective, this study presents an early application of a major, new, commercially available database populated from federal and some commercial sources. The for-profit company behind this database prides itself in "building the largest online healthcare data and information repository."4 Of course, a large administrative database can offer valuable insights into population-based questions. However, foundational concerns of transparency, accountability, and validation of a commercially based administrative claims database and its analytical tools will need to be addressed soon by the academic community as undoubtedly more research efforts will seek to use this convenient resource and eventually health-care policy decisions will be based upon resultant publications. To date, we at EBSJ could not find references to any peer-reviewed study attesting to the validity of this commercial database and its data gathering and analysis processes including from the company Web site itself. To be clear, we found no reason to disbelieve any of the data reports of this company. However, the very principle of a commercial enterprise needing to rely on proprietary tools in the realms of data gathering and analysis will likely make independent external validation difficult to come by. In an age where there is increasing pressure on researchers to be transparent, such validation will become increasingly important. EBSJ simply wishes to point out this dilemma as there is an increasing market for health-care databases and analytical services.

Furthermore, interesting questions are bound to be raised by such super-sized databases. The question of statistical significance becomes a relevant consideration. Simply by virtue of their size, it is possible that any differences in the studied populations may be statistically significant. Such differences may not be clinically significant and the magni- tude of effect may be small. As described in previous EBSJ "Science in Spine" articles, reliance on statistical significance without consideration of effect size, clinical relevance and potential for confounding, and other biases is ill-advised. The quality of any results from data mining depends on the quality of the data (again, validation of data is important). Exhaustive mining of data from such databases may produce results that are the product of random fluctuations and the relationships identified may be spurious. ${ }^{5-7}$

Aside from the relevance concern this raises, the other concern is that of the prospect of using mining of large databases for "dredging" or "fishing," which is random trawling through data in the hope of identifying associations or patterns. There is agreement in the scientific community that this is not an appropriate approach to answer important clinical research questions. A hypothesis-driven approach is for many reasons the gold standard and should remain such for important clinical questions. As with any quality study, focused, answerable questions developed a priori should guide the process and sound methods that address heterogeneity of data sources and pervasiveness of missing data followed. ${ }^{8}$ These are again simply abstract considerations to be pondered by the EBSJ readership and the scientific community at large. That said, the sheer scope of data provided in this article paired with a very important and sensible research question makes this a very relevant and noteworthy study and the editors strongly encouraged publication and congratulate the authors on their idea and study execution.

\section{References}

1 Ames CP, Blondel B, Scheer JK, et al. Cervical radiographical alignment: comprehensive assessment techniques and potential importance in cervical myelopathy. Spine (Phila Pa 1976) 2013; 38(22, Suppl 1):S149-S160

2 Ha Y, Schwab F, Lafage V, et al. Reciprocal changes in cervical spine alignment after corrective thoracolumbar deformity surgery. Eur Spine J 2013; October 18 (Epub ahead of print)

3 Smith JS, Lafage V, Ryan DJ, et al. Association of myelopathy scores with cervical sagittal balance and normalized spinal cord volume: analysis of 56 preoperative cases from the AOSpine North America Myelopathy study. Spine (Phila Pa 1976) 2013;38(22, Suppl 1): S161-S170

4 PearlDiver. Available at: http://www.pearldiverinc.com/about-us. php. Accessed December 9, 2013

5 Chopoorian JA, Witherell R, Khalil OEM, Ahmed M. Mind your own business by mining your data. SAM Advanced Management Journal 2001;66(2):45-51

6 Hand DJ. Data mining: statistics and more? Am Stat 1998;52(2): 112-118

7 Koh HC, Tan G. Data mining applications in healthcare.J Healthc Inf Manag 2005;19(2):64-72

8 Cios KJ, Moore GW. Uniqueness of medical data mining. Artif Intell Med 2002;26(1-2):1-24 